\title{
Study on damage evolution characteristics of coal and rock uniaxial compression based on the acoustic emission
}

\author{
Shugang $\mathrm{Li}^{1,}$, , Ning Wang ${ }^{1, b}$, Tianjun Zhang ${ }^{2, \mathrm{c}}$ \\ ${ }^{1}$ College of Energy Science and Engineering, Xi'an University of Science \& Technology, Xi'an, \\ Shaanxi, 710054, China \\ ${ }^{2}$ School of Science, Xi'an University of Science \& Technology, Xi'an, Shaanxi, 710054, China \\ alisg@.xust.edu.cn, bwangningxust@163.com, 'tianjun_zhang@126.com
}

\begin{abstract}
Keywords: Coal and Rock, Damage Evolution, Acoustic Emission, Crack Propagation.
Abstract. In order to obtain damage evolution characteristics of coal and rock under the compression, conduct uniaxial compression experiment for coal and rock with rigid loading experiment machine based on the acoustic emission. Gather the AE characteristic parameters in the process of coal and rock damage, use the damage variable of the accumulated $\mathrm{AE}$ ringing count, build uniaxial damage model for coal and rock based on the gathered acoustic emission characteristics, the damage evolution equation of coal and rock under compression is given, discuss the relationship between Acoustic emission parameters and mechanics failure mechanism of coal and rock. Research results show that acoustic emission signal can reflect the internal damage of coal and rock, which is closely related to their internal native crack compaction and evolution process including new crack production, extension and cut-through. The deformation and damage evolution degree of loaded coal and rock present positive correlation characteristics with accumulated acoustic emission ringing count.
\end{abstract}

\section{Introduction}

Uniaxial compression test results of coal and rock show that loading coal and rock damage presents the time effect, coal and rock deformation and failure can be seen as a gradual development process, which includes the deformation, damage initiation and evolution, and even a macroscopic crack, again by the whole process of crack propagation to destroy [1-3]. With Outer loads, the formation and extension of coal and rock internal micro defects, and their interaction jointly result in the macroscopic deformation and fracture characteristics of coal and rock $[4,5]$. Many scholars have done a large amount of basic research work in the failure process of coal and rock acoustic emission characteristics [6-8]. Research shows that, through the analysis and research of coal and rock acoustic emission signal, the inside of the coal and rock damage can be concluded and then the damage evolution can be studied [9-11].

In order to establish the relationship between parameters of acoustic emission and mechanics damage mechanism of coal and rock, get a better understanding of the damage evolution law of coal and rock, further reveal the dynamic disasters evolution process of coal and rock and disaster time effect mechanism, deformation damage of coal and rock and acoustic emission characteristics under uniaxial compression load are studied.

\section{Acoustic Emission Characteristics of the Coal and Rock under Uniaxial Compression}

Test loading system is electronic universal testing machine SANS30, acoustic emission measurement is conducted by the SDAES-5 acoustic emission detection system, as shown in Fig. 1. 


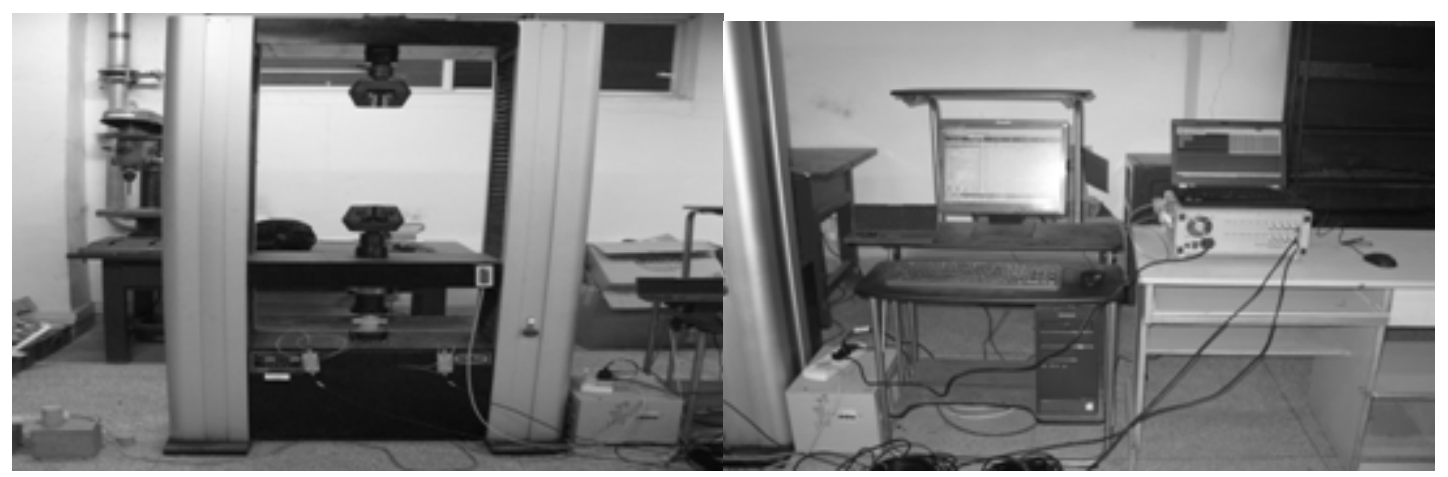

(a) Electronic Universal Testing Machine SANS30

(b) SDAES-5 Acoustic Emission Detection System

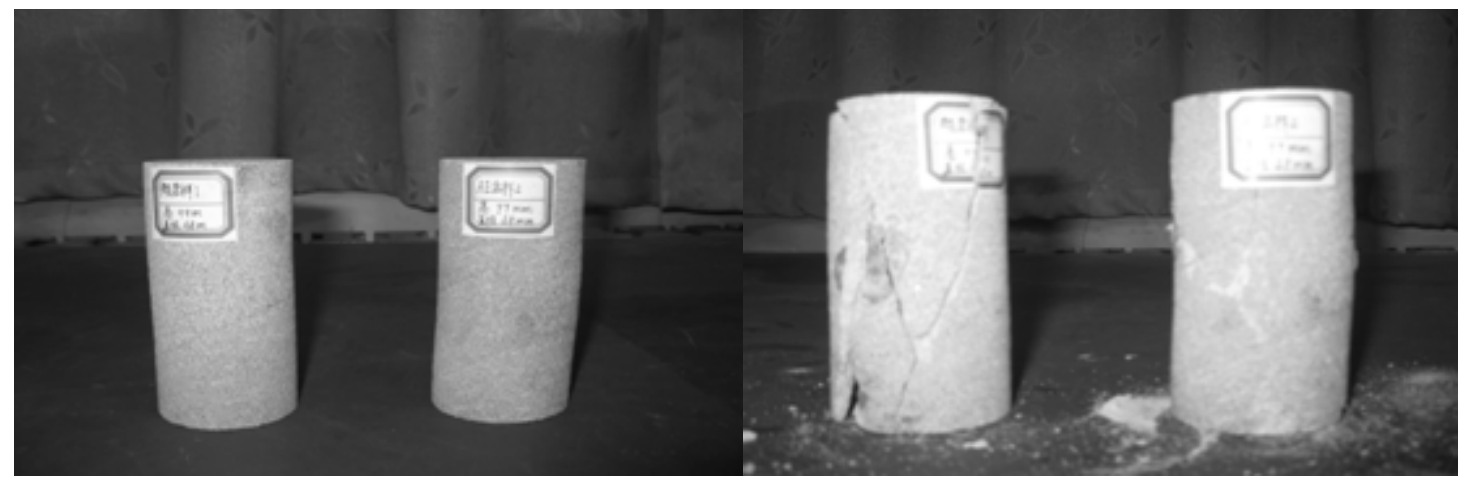

(c) Experimental Sample before Loading

(d) Breakage Sample after Loading

Fig. 1 Test Loading System and Test Sample

Coal and rock damage deformation and acoustic emission characteristic are tested under uniaxial compression condition, monitor acoustic emission signal, loading, deformation and time parameters in the deformation and failure process of coal and rock. Fig. 2, Fig. 3 show deformation and acoustic emission characteristics of typical coal and rock under uniaxial compression loading.

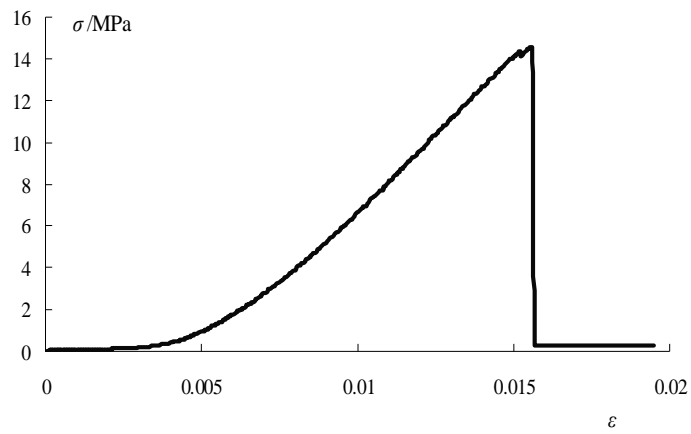

(a) Rock Sample R-1

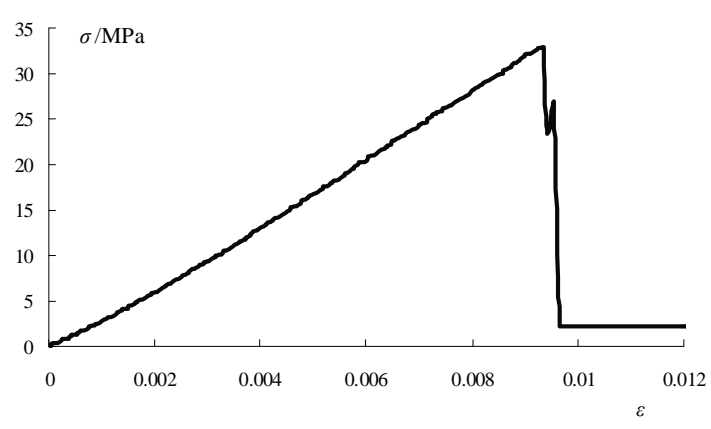

(b) Rock Sample R-2

Fig. 2 Coal and Rock Axial Stress-strain Curve under Uniaxial Compression Load

From Fig. 2, coal and rock axial stress-strain process curve under uniaxial compression can be divided into four stages including Compaction, elastic deformation, strain strengthen and strain flow. 


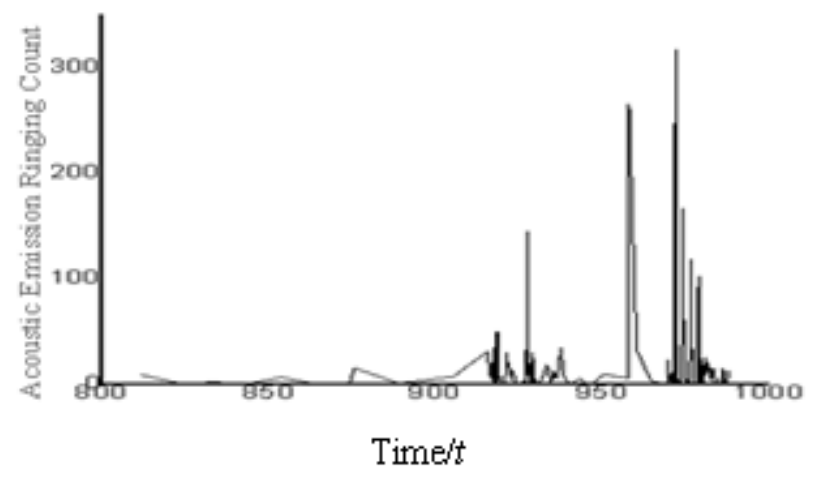

Fig. 3 Relation Curve of Acoustic Emission Accumulated Ringing Count and Time

From Fig. 3, there is less or even no acoustic emission ringing count in the earlier stages of loading, the initial cracks in the coal and rock begin to close, and the closing process, the closing part of the rough surface damage and closure crack surface slipping produce a small number of acoustic emission with less energy. With the slow increase of loads, cracks begin to expand, new cracks begin to produce, acoustic emission ringing count, cumulative ringing count and energy gradually tend to be more active. Continue to load, the interaction between cracks begin to increase, micro cracks occuring polymerization and penetration gradually form the macroscopic cracks. Near the peak stress, acoustic emission activities abnormally, and acoustic emission ringing counts reach to maximum in the peak stress. From the coal and rock specimen test curve, rock samples occur local failure, acoustic emission ringing count and energy have an obvious amplification after loading to $16 \mathrm{MPa}$.

\section{Coal and Rock Damage Model under Uniaxial Compression}

Ringing count is proportional to the strain energy releasing from coal and rock fracture and crack propagation, which is one of the parameters well to describe the characteristics of the material performance change, choose ringing count and cumulative ringing count to describe coal and rock damage characteristics. Damage variable is defined as Eq. 1:

$$
\mathrm{B}=\frac{\mathrm{A}_{b}}{\mathrm{~A}}
$$

In Eq. 1, $\mathrm{A}_{b}$ stands for all the area of micro defects on the cross section, $A$ means the sectional area of initial condition.

If the whole cross section $A$ of nondestructive material complete damage cumulative $\mathrm{AE}$ counts ringing is $C_{l}$, the acoustic emission ringing count while the micro unit of per unit area damaging $C_{w}$ is denoted as Eq. 2:

$$
C_{w}=\frac{C_{l}}{\mathrm{~A}}
$$

When the section damage area reaches $\mathrm{A}_{b}$, accumulated acoustic emission ringing count $C_{b}$ is shown in Eq. 3:

$$
C_{b}=\mathrm{C}_{w} A_{b}=\frac{C_{l}}{A} A_{b}
$$

Thus, damage variable is converted as Eq. 4:

$$
B=\frac{C_{b}}{C_{l}}
$$

In the test process, owing to lack of testing machine stiffness or setting different coal and rock damage conditions, coal and rock often haven't completely destroyed, that is, the coal and rock damage variable hasn't reached 1, the testing machine has stopped. Thus, damage variable is revised as Eq. 5:

$$
B=B_{s} \frac{C_{b}}{C_{l}}
$$


In Eq. 5, $B_{s}$ stands for damage threshold, and the values of $C_{l}$ is the accumulation of acoustic emission ringing count when damage variable reaches $B_{s}$. In order to simplify calculation, the value of damage threshold $B_{s}$ is defined as Eq. 6:

$$
B_{s}=1-\frac{\sigma_{c}}{\sigma_{p}}
$$

In Eq. $6, \sigma_{p}$ is peak intensity, $\sigma_{c}$ is residual strength. Thus, damage model of coal and rock under uniaxial compression based on the acoustic emission characteristics is shown as Eq. 7:

$$
\sigma=(1-D) E \varepsilon=\left(1-B_{S} \frac{C_{b}}{C_{l}}\right) E \varepsilon
$$

\section{Damage Evolution Analysis of Coal and Rock under Uniaxial Compression}

According to the damage variable of accumulated acoustic emission ringing count in Eq. 5, use Eq. 6 to fit strain-coal and rock damage parameters resulting in $\sigma_{c}=2.06 \mathrm{MPa}$ and $\sigma_{p}=11.93 \mathrm{MPa}$. These results near the compression test ones, which shows that the damage variable defined in Eq. 5 of accumulated acoustic emission ringing count is reasonable.

\section{Conclusions}

Using SANS30 loading system and SDAES-5 acoustic emission detection system, experimentally study the damage evolution of coal and rock under uniaxial compression and acoustic emission properties. The results are as follows:

Use the damage variable of accumulated acoustic emission ringing count, establish the relationship between acoustic emission parameter and the mechanics failure mechanism of coal and rock, and verify its rationality.

Build damage model of coal and rock under uniaxial compression based on acoustic emission characteristics, obtain damage evolution curve and equation of coal and rock, lay the foundation to better understand the damage evolution law of coal and rock and to further reveal the dynamic disasters evolution process and disaster time effect mechanism of coal and rock.

Acoustic emission information reflects the internal damage of coal and rock, which is closely related to its internal native fracture compression and evolution process including new crack produce, extension and transfixion. There is less or even no acoustic emission ringing count in the earlier stages of loading, the initial cracks in the coal and rock begin to close, and the closing process, the closing part of the rough surface damage and closure crack surface slipping produce a small number of acoustic emission with less energy. With the slow increase of loads, cracks begin to expand, new cracks begin to produce, acoustic emission ringing count, cumulative ringing count and energy gradually tend to be more active. Continue to load, the interaction between cracks begin to increase, micro cracks occuring polymerization and penetration gradually form the macroscopic cracks. Near the peak stress, acoustic emission activities abnormally, and acoustic emission ringing counts reach to maximum in the peak stress.

\section{Acknowledgements}

This work was carried out in the context of the Project NO. 51327007, 51174157, 51374168 supported by the National Natural Science Foundation of China (NSFC). The financial support is greatly acknowledged. 


\section{References}

[1] LI Huigui, GAO Baobin, LI Huamin. Study on Macroscopic Fracture Structure and Acoustic Emission Character of Coal Rock under Uniaxial Compression[J]. Chinese Journal of Underground Space and Engineering, 11(3): 612-618. (2015)

[2] JI Honggunag, LU Xiang. Characteristics of Acoustic Emission and Rock Fracture Precursors of Granite under Conventional Triaxial Compression[J]. Chinese Journal of Rock Mechanics and Engineering, 34(4): 694-702. (2015)

[3] ZHANG Liming, MA Shaoqiong, REN Mingyuan, et al. Acoustic Emission Frequency and $b$ Value Characteristics in Rock Failure Process under Various Confining Pressures[J]. Chinese Journal of Rock Mechanics and Engineering, 34(10): 2057-2063. (2015)

[4] ZHANG Zhaopeng, ZHANG Ru, ZHANG Zetian, et al. Experimental Research on Effects of Bedding Plane on Coal Acoustic Emission under Uniaxial Compression[J]. Chinese Journal of Rock Mechanics and Engineering, 34(4): 770-778. (2015)

[5] YANG Yongjie, WANG Dechao, LI Bo, et al. Acoustic Emission Characteristics of Coal Damage Failure under Triaxial Compression[J]. Journal of Basic Science and Engineering, 23(1): 127-135. (2015)

[6] WANG Chao, YANG Xiaobin, GUO Weiqi, et al. Uniaxial Loading Statistical Damage Constitutive Model for Coal and Rock[J]. Safety in Coal Mines, 46(11): 10-13. (2015)

[7] GU Yilei, WU Wenjie, LI Qingmiao, et al. Acoustic Emission Characteristics of Rock during Whole Stress-strain and Unloading Process under Different Confinong Pressure[J]. Safety in Coal Mines, 46(6): 36-39. (2015)

[8] XIE Yong, HE Wen, ZHU Zhicheng, et al. Study on Backfill Acoustic Emission Characteristic and Damage Evolution under Uniaxial Compression[J]. Chinese Journal of Applied Mechanics, 32(4): 670-676. (2015)

[9] CAO Anye, JING Guangcheng, DOU Linming, et al. Damage Evolution Law Based on Acoustic Emission of Sandy Mudstone under Different Uniaxial Loading Rate[J]. Journal of Mining \& Safety Engineering, 32(6): 923-935. (2015)

[10]NIU Shuangjian, FENG Wenlin, DANG Yuanbeng, et al. Experimental Study of Acoustic Emission and Energy Dissipation Characteristics of Cracked Rock Subjected to Uniaxial Compression[J]. Journal of Experimental Mechanics, 30(4): 515-522. (2015)

[11] ZHAO Xiaopng, CHEN Shufen. Failure Evolution Process of Fractured Rockmass Based on Amplitude of Acoustic Emission[J]. hinese Journal of Rock Mechanics and Engineering, 34(1): 3012-3017. (2015) 